\title{
Grimes on the tacking by disjunction problem
}

\author{
Luca Moretti \\ King's College London
}

\begin{abstract}
In this paper, I focus on the so-called 'tacking by disjunction problem.' Namely, the problem to the effect that, if a hypothesis $h$ is confirmed by a statement $e, h$ is confirmed by the disjunction $e \vee f$, for whatever statement $f$. I show that the attempt to settle this difficulty made by Grimes 1990, in a paper apparently forgotten by today methodologists, is irremediably faulty.
\end{abstract}

A simple formulation of Hypothetico-deductivism states that, if $h$ is a hypothesis and $e$ an evidential statement:

(HD) $e$ confirms $h$ if (1) $e$ is true, (2) $h$ and $\sim e$ are each consistent, and (3) $h$ $\vdash e$ [i.e. $h$ entails $e$ ]. ${ }^{1}$ (Grimes 1990, 520).

(HD) implies the following rule:

(TDP) If $e$ confirms $h$ in accordance with (HD), then the disjunction $e \vee f$ will also confirm $h$ where $f$ is any arbitrary statement that is not entailed by $\sim e$. (Ibid.)

Consider in fact that, if $e$ confirms $h$ in accordance with (HD), $h$ entails $e$, thus, by the rule of the introduction of disjunction, (3) $h$ entails $e \vee f$ too. Moreover, if $e$ confirms $h$ in accordance with (HD) and $f$ is not entailed by $\sim e$, (2) $h$ and $\sim(e \vee f)$ prove each consistent. Thus, if (1) $e \vee f$ is true, in accordance with (HD), $e \vee f$ confirms $h$.

(TDP) is problematic, first of all, because it can make h's confirmation depend on the verification of statements that are intuitively irrelevant for

\footnotetext{
${ }^{1}$ Notice that, if (2) $h$ and $\sim_{e}$ are each consistent and (3) $h$ entails $e$, then $h$
} and $e$ must be contingent. This makes them liable to confirm and to be confirmed. 
such a confirmation. Let us suppose, for example, that $h$ is Newton's Mechanics, $e$ is its consequence that a certain body $A$, if dropped, falls in accordance with the Free-Fall Law, and $f$ is the statement "my name is John.' In this case, since $e$ confirms $h$ in accordance with (HD) and $f$ is not entailed by $\sim e$, on the grounds of (TDP), $e \vee f$ confirms $h$. Consider now the case in which $e$ has not been verified and my name is in fact John. Because of the truth of $f, e \vee f$ is true too. Thus, given (TDP), Newton's Mechanics is confirmed by the fact that my name is John, which is hardly acceptable.

Indeed, there are even more counterintuitive cases of confirmation allowed by (TDP). Let us suppose that $h$-i.e. Newton's Mechanics entails two statements $e_{1}$ and $e_{2}$, where both of them confirm $h$ in accordance with (HP). $e_{1}$ says again that the body $A$ falls in accordance with the Free-Fall Law and $e_{2}$ says that another body $B$ falls the same way. Consider now the disjunction $e_{1} \vee \sim e_{2}$. Since $\sim e_{2}$ is not entailed by $\sim_{e_{1}}$ and $e_{1}$ confirms $h$ in accordance with (HD), given (TDP), the disjunction $e \vee \sim_{e_{2}}$ will confirm $h$ too. This means that, if $\sim_{e_{2}}$ is true, $h$ is confirmed. But this is just absurd, as $h$ entails the logical negation of $\sim e_{2}$.

Grimes believes that 'the basic idea underlying the $[\ldots$ hypotheticodeductive] method is that a hypothesis is confirmed if part of its content, part of what it asserts about the world, is shown to be true.' (Grimes $1990,517)$. On the other hand, according to him, in the paradoxical confirmation cases allowed by (HD), 'though $h$ will entail the disjunction $e$ $\vee f$ if $h$ entails $e$, the disjunction seems not to be part of the content of $h$.' (Grimes 1990, 520). Grimes is thus persuaded that the tacking by disjunction problem can be settled if (HD) is replaced by a rule closer to the basic hypothetico-deductivist idea that a hypothesis is confirmed if part of its content is shown to be true.

To articulate his solution, Grimes formulates Hypotheticodeductivism by resorting to the notion of a disjunction in Boolean normal form and the notion of a narrow consequence. As Grimes explains:

Each disjunct of a disjunction in Boolean normal form consists of a conjunction which includes in alphabetical order exactly one occurrence of each atomic expression or its negation (but not both) that occurs in the larger disjunction, where no two disjuncts are the same. For example, $(p \& q) \vee(\sim p \&$ $q) \vee(\sim p \& \sim q)$ is a disjunction in Boolean normal form that is equivalent to the conditional $p \supset q$. (Grimes 1990, footnote 4, 520). 
Grimes then defines the notion of a narrow consequence, represented by the symbol ' $\mapsto$ ', as follows:

$p \mapsto q={ }_{d f} p$ and $\sim q$ are each consistent and there is a disjunction $d_{1} \vee \ldots \vee d_{k}$ in Boolean normal form such that (i) $\vdash q \equiv\left(d_{1} \vee \ldots \vee d_{k}\right)$, and (ii) for some $d_{\mathrm{i}}, p \vdash d_{\mathrm{i}}$. (Grimes 1990, 520).

According to Grimes, 'the notion of a narrow consequence seems better suited [than that of a logical consequence] for expressing a relation of preserving content.' (Grimes 1990, 520).

Eventually, Grimes formulates Hypothetico-deductivism in terms of this principle:

(HD1) $e$ confirms $h$ if (1) $e$ is true, (2) $h$ and $\sim e$ are each consistent, and (3) $h \mapsto e .^{2}$ (Ibid.)

Grimes' solution of the tacking by disjunction problem is apparently efficacious. Let us suppose, for instance, that $d, e$ and $f$ are atomic expressions, that $h \equiv_{d f} d \& e$, and that $h$ and $\sim(e \vee f)$ are each consistent. Notice that, in this case, the verification of $f$ is surely irrelevant for $h$ 's confirmation. Since $h$ entails $e$ and $e$ entails $e \vee f$, h entails $e \vee f$ too. However, this disjunction is not a narrow consequence of $h$. For $e \vee f$ is equivalent to the disjunction in Boolean form $(e \& f) \vee(\sim e \& f) \vee(e \& \sim f)$, where $h$ entails none of these disjuncts. Since the requirement (3) of (HD1) is not fulfilled, $e \vee f$ does not confirm $h$. More generally, Grimes' solution depends on the fact that the rule of the introduction of the disjunction does not hold on the notion of strict consequence. Thus, if $h \mapsto e$, it is not true that, for every $f, h \mapsto e \vee f$.

Grimes' proposal has surely the merit of being very intuitive, simple and elegant. If it worked, it could probably be preferred to other attempted solutions available in literature now, which are more complex. ${ }^{3}$ Unfortunately, Grimes' solution is faulty. The trouble with (HD1) is that, though it does not entail the problematic rule (TDP), it cannot dismiss at

${ }^{2}$ If (2) $h$ and $\sim_{e}$ are each consistent and (3) $e$ is a strict consequence of $h$ (thus $h$ entails $e$ ), $h$ and $e$ are contingent and liable to confirm and to be confirmed.

${ }^{3}$ See for instance Kuipers (2000, 17-27).

${ }^{4}$ Unnoticed in the substantially positive review of Grimes' paper made by Skyrms 1992. 
least some of the most counterintuitive confirmation cases, allowed by (TDP), that I have discussed above. (HD1) in fact entails that:

(TDP1) If $e_{1}$ and $e_{2}$ are atomic sentences that confirm $h$ in accordance with (HD1), then the disjunction $e_{1} \vee \sim_{e_{2}}$ will also confirm $h$ if $\sim_{e_{2}}$ is not entailed by $\sim e_{1} .{ }^{5}$

(HD1) implies (TDP1) because $e_{1} \vee \sim e_{2}$ is equivalent to the disjunction in Boolean normal form $\left(e_{1} \& \sim e_{2}\right) \vee\left(\sim e_{1} \& \sim e_{2}\right) \vee\left(e_{1} \& e_{2}\right)$, where the last disjunct is entailed by $h$. For $h$ implies, by assumption, both $e_{1}$ and $e_{2}{ }^{6}$

The problems affecting Grimes' solution are indeed more serious. For the notion of a narrow consequence of a theory is meant, by Grimes, to express 'a relation of preserving content', but it is evident that it does not do. The reason is that the disjunction $e_{1} \vee \sim e_{2}$ and other statements one can construct at one's will, like $\left(\sim e_{1} \& e_{2}\right) \vee\left(e_{1} \& \sim e_{2}\right) \vee\left(e_{1} \& e_{2}\right)$ and $\left(\sim e_{1}\right.$ $\left.\& \sim_{e_{2}}\right) \vee\left(e_{1} \& e_{2}\right),{ }^{7}$ which are clearly not part of h's content, prove all strict consequences of $h$. Notice finally that all these statements do confirm $h$ in accordance with (HD1). Thus, for instance, on the grounds of $\left(\sim e_{1} \& \sim e_{2}\right) \vee\left(e_{1} \& e_{2}\right)$, whenever $\sim e_{1} \& \sim e_{2}$ is verified, $h$ is confirmed, which is completely unacceptable.

A seemingly promising strategy to settle such difficulties might be that of re-defining the notion of a strict consequence to the effect that $q$ strictly follows from $p$ if and only if both $p$ and $\sim q$ are consistent and $p$ entails each disjunct (individually taken) of the disjunction in Boolean normal form equivalent to $q$. Notice in fact that, if $h$ entails $e_{1}$ and $e_{2}, h$ does not entail each disjunct of the disjunction in Boolean form equivalent to $e_{1} \vee \sim e_{2}$; the same happens in all other unwanted confirmation cases considered above. Unfortunately, this easy loophole does not work, as so defined a notion of a strict consequence is actually too strict. This notion appears to fit well intuitive content relations between simple

${ }^{5}$ Probably, (TDP1) could be re-formulated to include couples of statements $e_{1}$ and $e_{2}$ that are not atomic. The present formulation is however sufficient to show the flaws in Grimes' solution.

${ }^{6}$ More exactly, since $h$ implies one of those disjuncts, (3) $e_{1} \vee \sim_{e_{2}}$ is a strict consequence of $h$. Moreover, since $e_{1}$ confirms $h$ in accordance with (HD1) and $\sim_{e_{2}}$ is not entailed by $\sim_{e_{1}},(2) h$ and $\sim\left(e_{1} \vee \sim_{e_{2}}\right)$ prove each consistent. Thus, when (1) $e_{1} \vee \sim_{e_{2}}$ is true, in accordance with (HD1), $e_{1} \vee \sim_{e_{2}}$ confirms $h$. form.

${ }^{7}$ Notice that all these statements are already disjunctions in Boolean normal 
logical conjunctions. For example, it is intuitive that $P a \& Q a$ is part of the content of $\forall x\left(P_{x} \& Q x\right)$ and, in this case, $\forall x\left(P_{x} \& Q x\right)$ entails just each disjunct of the disjunction in Boolean form equivalent to Pa \& Qa. Consider however the following two statements that are not logical conjunctions: $\forall x\left(P_{x} \supset Q_{x}\right)$ and $P_{a} \supset Q_{a}$. It is intuitive that $P_{a} \supset Q_{a}$ is part of the content of $\forall x\left(P_{x} \supset Q_{x}\right)$, and yet the latter does not entail each disjunct of the disjunction in Boolean form equivalent to the former. Further examples similar to the latter can easily be produced.

I do not see any immediate 'technical refinement' of Grimes' notion of a strict consequence or of (HD1) that could enable Grimes to overcome the difficulties I have discussed in this paper. My conclusion is that Grimes' solution of the tacking by disjunction problem is seriously flawed and it cannot be accepted.

Luca Moretti

Department of Philosophy

King's College London

Strand, London WC2R 2LS

luca.moretti@kcl.ac.uk

\section{References}

Grimes, Thomas R. 1990. Truth, Content, and the Hypothetico-Deductive Method. Philosophy of Science 57: 514-22.

Kuipers, Theo A. F. 2000. From Instrumentalism to Constructive Realism. Dordrecht: Kluwer Academic Publishers.

Skyrms, Brian. 1992. Review of Glymour 'Hypothetico-deductivism is hopeless', Waters 'Relevance Logic brings hope for hypotheticodeductivism' and Grimes 'Truth, Content and Hypothetico-deductive method'. The Journal of Symbolic Logic 57: 756-758. 